Revue interdisciplinaire des études canadiennes en

France

$87 \mid 2019$

Solitude(s) au Canada

\title{
La « Troisième Solitude » du Canada vécue et transcrite par deux écrivaines autochtones : Jeannette Armstrong et Maria Campbell
}

Canada's Third Solitude as seen and transmitted by two Native women writers, Jeannette Armstrong and Maria Campbell

Isabelle Eloy-Carriat

\section{(2) OpenEdition}

\section{Journals}

Édition électronique

URL : https://journals.openedition.org/eccs/3019

DOI : $10.4000 /$ eccs.3019

ISSN : 2429-4667

Éditeur

Association française des études canadiennes (AFEC)

Édition imprimée

Date de publication : 4 décembre 2019

Pagination : 87-105

ISSN : 0153-1700

Référence électronique

Isabelle Eloy-Carriat, « La « Troisième Solitude » du Canada vécue et transcrite par deux écrivaines autochtones : Jeannette Armstrong et Maria Campbell », Études canadiennes / Canadian Studies [En ligne], 87 | 2019, mis en ligne le 01 décembre 2020, consulté le 18 juin 2021. URL : http:// journals.openedition.org/eccs/3019; DOI : https://doi.org/10.4000/eccs.3019 


\title{
La «Troisième Solitude » du Canada vécue et transcrite par deux écrivaines autochtones : Jeannette Armstrong et Maria Campbell
}

\author{
Isabelle ELOY-CARRIAT, Université de Rouen Normandie
}

\begin{abstract}
Nous nous intéresserons aux écrivains autochtones, en limitant notre étude à deux écrivaines autochtones canadiennes, Jeannette Armstrong et Maria Campbell. Isolées à double titre en tant que femmes et Autochtones, elles tenteront de briser « cette solitude », solitude intensifiée par le métissage pour Maria Campbell. Après l'expérience du militantisme et de la transmission de leurs valeurs, elles font l'expérience d'une forme de thérapie à travers l'affirmation de leur identité et elles œuvrent à la réconciliation de tout un peuple, d'abord au sein de leurs propres nations, et dans un second temps avec la société d'origine européenne.

Our study focuses on Indigenous writers, namely on two Indigenous women writers: Jeannette Armstrong and Maria Campbell. Isolated both as women and Indigenous persons, they try to break their respective sense of solitude, a solitude which is increased by interbreeding for Maria Campbell. After being involved in activism and the transmission of their values, they discover a form of therapy through the assertion of their identity and they aim at the reconciliation of a whole people, first within their own nations and then with society of European descent.
\end{abstract}

Au Canada, les peuples autochtones ${ }^{1}$ sont souvent désignés comme étant la «Troisième Solitude $»^{2}$, après ces deux autres solitudes que sont les Canadiens francophones et les Canadiens anglophones. Je m'intéresserai au sentiment de solitude des Autochtones ressenti de différentes façons, par une femme membre d'une Première Nation et par une autre, qui vit l'expérience du métissage. Puis, j'approfondirai l'étude de ce sentiment, encore plus exacerbé si l'Autochtone en question est une femme, car les femmes, toutes origines confondues, sont encore bien souvent victimes de discrimination dans notre monde occidental, et les femmes autochtones souffrent du système patriarcal imposé à leurs sociétés. Ainsi, je m'appliquerai à démontrer comment deux écrivaines canadiennes, Jeannette Armstrong et Maria Campbell, isolées à double titre en tant que femmes et Autochtones, dénoncent cette solitude, solitude intensifiée par le métissage pour Maria Campbell. Si j'ai choisi de me pencher sur ces deux écrivaines en particulier, c'est parce qu'elles font partie des pionnières de l'autobiographie autochtone canadienne et que leur témoignage apporte un éclairage précieux sur la solitude particulière vécue par les femmes autochtones au Canada. Toutes deux

\footnotetext{
${ }^{1}$ La Constitution canadienne reconnaît trois groupes de peuples autochtones : les Indiens (aujourd'hui plus souvent appelés les « Premières Nations »), les Inuit et les Métis (Loi constitutionnelle de 1982, article 35).

${ }^{2}$ La genèse de cette expression n'est pas facile à déterminer. Il est probable que John Ralston Saul ait été le premier à l'employer, dans son ouvrage de 1997, Reflections of a Siamese Twin. Joseph Boyden, qui était proche de Saul, a dû reprendre le concept de lui et l'a beaucoup employé. C'est une expression qui est depuis passée dans le langage courant et les media, surtout depuis la Commission de vérité et réconciliation (2008-2015). L'auteure remercie Lorie-Anne Rainville pour ces aimables indications.
} 


\section{ISABELLE ELOY-CARRIAT}

font l'expérience de la tension créée par la nécessité de vivre dans une culture blanche dominante tout en préservant les aspects essentiels de sa propre communauté autochtone, et en rendent compte à travers leurs ouvrages respectifs, Slash et Halfbreed.

\section{Préoccupations des communautés minoritaires fondatrices canadiennes face à la sauvegarde de leur identité culturelle}

La marginalisation sociale, économique et politique, la discrimination n'ont concédé aux peuples autochtones du Canada qu'un pouvoir social et politique minime. Encore aujourd'hui, le manque d'éducation, d'emplois, de compétences, de possibilités et d'équité salariale sévit dans plusieurs communautés autochtones, aussi bien urbaines que rurales.

N'est-ce pas une ironie de l'histoire que des préjugés de soi-disant «infériorité » soient endurés par les peuples autochtones d'un pays qui s'est construit à travers l'immigration d'Européens arrivés pendant tout le XVII ${ }^{\text {ème }}$ siècle et une grande partie du XVIII ${ }^{\text {ème }}$, donc des étrangers à cette terre d'accueil ? Maria Campbell retrace ainsi l'histoire des Métis : «The Halfbreeds then became squatters on their land » (CAMPBELL 1973, 13).

On distinguera deux formes de solitude différentes mais complémentaires endurées par une Okanagane et par une Crie-Métisse ${ }^{3}$. La solitude vécue et transcrite par Jeannette Armstrong est la solitude d'une minorité conquise par le colonisateur qui devient rapidement oppresseur. Outre la perte de leurs territoires au cours du XIX ${ }^{\mathrm{ème}}$ siècle, les peuples autochtones se sont trouvés dépossédés de leur mode de vie traditionnel et de leur autonomie. C'est un isolement géographique, dans les réserves : un peuple fier et autonome se réduit à être assisté après avoir été spolié de son territoire.

Les Métis se sont toujours considérés comme un peuple à part. Néanmoins, qu'ils soient membres des Premières Nations ou métis, les Autochtones subissent la violence de mesures visant à les assimiler en gommant les différences. Ainsi l'enseignement de l'anglais est imposé aux enfants dès 1950, avec interdiction de parler leur langue ${ }^{4}$. Ceci les incite à rejeter les langues

\footnotetext{
${ }^{3}$ Le père de Maria Campbell était le petit-fils d'un homme d'affaires écossais et d'une Métisse en partie Crie ; sa mère était la fille d'une Crie et d'un Franco-Américain. Présentation en ligne de Halfbreed (Penguin Random House, édition de poche, 2019) https://www.penguinrandomhouse.com/books/610927/halfbreed-by-maria-campbell/

${ }^{4} \mathrm{La}$ langue est un enjeu et par conséquent elle est au centre de relations qui sont ipso facto marquées par le pouvoir. L'interdiction de parler sa langue se rencontre dans de nombreux endroits sur tous les continents quand le pouvoir en place veut imposer la langue du colonisateur.
} 


\section{LA « TROISIÈME SOLITUDE » DU CANADA VÉCUE ET TRANSCRITE PAR DEUX ÉCRIVAINES AUTOCHTONES : JEANNETTE ARMSTRONG ET MARIA CAMPBELL}

autochtones, même dans le cercle familial. Cet interdit qui touche au langage, à l'expression de soi et des sentiments les plus profonds, les plonge dans la confusion et l'incertitude. En effet ces générations-là ont dû apprendre l'anglais. Bien qu'en 2016, on dénombre encore plus de 70 langues autochtones au Canada (STATISTIQUE CANADA 2016), les jeunes Autochtones oublient la façon de vivre de leurs ancêtres : «All the people taking welfare and all the people drinking and forgetting how to ranch and hunt » (ARMSTRONG 1985, 28). Si culture, croyances et rites sont bafoués, alors ce peuple souffre de la perte de son identité.

George Ryga, dans sa préface à Slash qui date de 1984, porte une accusation cinglante contre le gouvernement canadien: «war and genocide against aboriginal peoples by corporations and government agencies protecting such practices through deliberately corrupt legislation and abuses of power » (ARMSTRONG 1985, 12). Le protagoniste Slash fait référence, non sans quelque amertume, à cette volonté de génocide lorsqu'il arrive à un powwow dans le Montana, dans les années 1970 : «Somehow it was comforting to know we were a long way from total extinction » (ARMSTRONG 1985, 90). Néanmoins, même si les Autochtones ont survécu, la diminution de leur population ne fait qu'accroître le sentiment de solitude. Les ravages de l'alcool achèvent la décimation que les épidémies avaient déjà causée : ainsi, lors du recensement de 2016, seulement 4,9\% de la population totale du Canada s'identifie comme autochtone ${ }^{5}$.

Dans les années 1970, les écoles mixtes sont créées par le Ministère des Affaires Indiennes pour intégrer les Autochtones à la population blanche, mais les mentalités n'ont pas évolué et Slash, élève plutôt doué, se sent rapidement exclu, méprisé et écarté, non seulement par les élèves mais encore par les professeurs. Slash relate la discrimination dont souffrent les jeunes Autochtones scolarisés dans les villes : «Our people seem to be looked on as if we were less instead of just different »(ARMSTRONG 1985, 87).

Deux systèmes de valeurs entrent alors en conflit : « So confusion arises inside each of the Indian kids who begin to question which value system they must live by » (ARMSTRONG 1985, 21). En effet, si l'enfant autochtone se met à rejeter sa culture, son mode de vie, ses rites, ce sont ses propres parents qu'il finit par rejeter. Quant à Maria Campbell, elle subit aussi de plein fouet la ségrégation à l'école : une séparation spatiale et culturelle est prédéfinie entre le groupe européen et le groupe autochtone. Boris Cyrulnik démontre que la

\footnotetext{
5 1,6 million de personnes, réparties comme suit : 977230 membres des Premières Nations, 58745 Métis et 6525 Inuits (STATISTIQUE CANADA 2016).
} 


\section{ISABELLE ELOY-CARRIAT}

privation de rite empêche l'enfant de s'inscrire dans un milieu affectif et social et conclut : «On ne peut pas vivre sans culture » $(2012,90)$

Après une phase de rejet, Slash le jeune Okanagan forme le vœu, propre à tout être humain, d'être reconnu (voire «réhabilité »). Les femmes, quant à elles, dès les années 1970, jouissent d'une autonomie toute relative. Elles ont longtemps été écartées de la culture. Dans la sphère de l'écriture, les femmes qui cherchent à s'imposer comme écrivaines autochtones désirent la reconnaissance d'une écriture intrinsèquement féminine. Or, elles se sentent incomprises et ressentent donc une solitude à la fois culturelle et matérielle. La société dans laquelle elles évoluent est de fait patriarcale: la femme y est infériorisée, abandonnée, marginalisée. De même en ce qui concerne les arts tels le cinéma et la littérature, l'image de la femme autochtone évolue au $X^{\text {ème }}$ siècle à travers le regard que l'Européen porte sur elle.

Cette problématique est reprise dans l'article de l'Encyclopédie canadienne sur la démographie des Autochtones :

Les problèmes auxquels font face les femmes autochtones sont principalement issus des préjugés à leur égard, perpétués par les hommes européens depuis leur arrivée en Amérique. [...] Comme les Autochtones sont évincés de leur territoire et placés dans des réserves [...] la frustration et la colère qui s'ensuivent se manifestent souvent dans la violence que les maris exercent sur leurs femmes (AYLSWORTH \& TROVATO 2015).

Dans Halfbreed, Maria Campbell évoque sa rencontre avec Stan Daniels, un Métis qui cherche à améliorer le sort des femmes autochtones : «He was really concerned about the plight of Native girls on the street [...] He understood how the Indian women felt about being abandoned by their men » (CAMPBELL 1973, 145).

Le même article de l'Encyclopédie canadienne évoque ainsi le sort des femmes autochtones :

Le rapport, publié en 2014, révèle qu'entre 1980 et 2012, jusqu'à 1181 femmes autochtones sont assassinées ou portées disparues. En date de septembre 2013, 164 femmes autochtones sont portées disparues, représentant $11,3 \%$ de toutes les femmes disparues au Canada. Puisque les femmes autochtones ne comptent que pour $4,3 \%$ de la population féminine totale du Canada, ces chiffres sont gravement disproportionnés. Plus effarant encore, 1017 meurtres de femmes autochtones ont été rapportés au cours de cette période, soit $16 \%$ du total des homicides de femmes dans tout le Canada (AYLSWORTH \& TROVATO 2015). 


\section{LA « TROISIÈME SOLITUDE » DU CANADA VÉCUE ET TRANSCRITE PAR DEUX ÉCRIVAINES AUTOCHTONES : JEANNETTE ARMSTRONG ET MARIA CAMPBELL}

C'est la raison pour laquelle, Okanagane ou Crie-Métisse, elles peinent à s'exprimer, voire à devenir le porte-parole de leur peuple ; la reconnaissance par les hommes autochtones (ou blancs) de leurs qualités d'écrivaines leur est refusée.

Dès les années 1970, la femme autochtone qu'est Maria Campbell désire ardemment parler en son nom propre et veut mettre fin à cette solitude. Elle veut être partie prenante d'un mouvement plus général de reconnaissance d'égalité des droits des femmes. Et pour y parvenir, elle doit se rapprocher des autres femmes autochtones, comme son arrière-grand-mère paternelle Cheechum le lui conseillait: «You have many sisters out there, my girl. You'll find them » (CAMPBELL 1973, 145).

L'importance du récit oral à l'origine, la transmission se faisant par le truchement des récits et légendes racontés par les femmes, explique l'accès tardif à l'écriture des femmes autochtones. L'ouvrage de Beverly Hungry Wolf (1982) l'illustre parfaitement grâce aux témoignages personnels de différentes femmes, sous forme de poésie ou de prose. Cet ouvrage offre une étude ethnologique de l'intérieur et non rapportée par un Européen. Il s'agit bien d'un désir de rompre la solitude de tout un peuple oublié en valorisant la culture autochtone, et d'affirmer avec détermination une identité collective.

C'est dans cette même optique que la femme autochtone a recours au récit autobiographique dès les années 1970. À l'époque, ces femmes écrivaines sont rares et isolées par rapport aux courants littéraires féminins contemporains. Si c'est la femme autochtone qui écrit, la rétrospective sera plus réaliste, le récit plus authentique - «to write about my life » (CAMPBELL 1973, 7) - que la biographie écrite par un écrivain blanc et européen, qu'il soit masculin ou féminin, qui va dénaturer le témoignage par son interprétation.

Maria Campbell se veut témoin fidèle de la vie des Autochtones. À la fin des années 1970, lorsqu'elle retourne sur son lieu de naissance, elle constate la dure réalité : « the streets were full of Native people in all stages of intoxication... The old angry bitter feeling came back to my stomach - the feeling of hate - as I saw people whom I had known as a child, now with such empty, despairing faces » (CAMPBELL 1973, 148). Elle met sa colère au service des autres et tente d'aider les filles qui vivent dans la rue en établissant un lieu où elles ont la possibilité de se réfugier, car elle veut les amener à découvrir leur identité. Sa démarche est finalement en accord avec la philosophie de Cheechum qui lui dit : «Each of us has to find himself in his own way and no one can do it for us ... The blanket only destroys, it doesn't give warmth » (CAMPBELL 1973, 150). 


\section{ISABELLE ELOY-CARRIAT}

Pour combattre son isolement, la femme autochtone se livre à une quête identitaire grâce à un retour au respect des origines et de l'héritage culturel (traditions, langue, coutumes). Le récit de Halfbreed s'achève en ces termes : «I have brothers and sisters, all over the country. I no longer need my blanket to survive » (CAMPBELL 1973, 157). Et dans Slash, le ton est donné dès le prologue: «I'm an Indian person» (ARMSTRONG 1985, 13). Nos deux antihéros affirment tour à tour leur identité en tant qu'autochtones.

\section{Un isolement vécu de manière différente par chaque écrivaine}

Il convient de distinguer les conditions dans lesquelles a grandi Jeannette Armstrong, Okanagane, de celles vécues par Maria Campbell, Crie-Métisse, tiraillée entre deux cultures et qui nous offre un récit plus intimiste.

En outre, la complexité de toute entreprise d'écriture quant à l'identité du sujet émerge ici, pour donner sa place à la distance entre sujet écrivant et narrateur et/ou personnage principal. Jeannette Armstrong, à travers son personnage Slash, relate son expérience de femme autochtone ayant grandi au sein de la Réserve des Okanagans en Colombie Britannique. Lorsque Slash s'exprime, c'est la voix de Jeannette Armstrong qu'il faut entendre, mais elle établit derechef une distance. Tout énoncé produit par un écrivain n'est pas à prendre pour argent comptant, et peut-être la démarche identitaire s'accomplitelle justement dans cet écart entre pensée intime et représentation publiée. Lorsque l'on lit Slash, et que l'on s'intéresse à la vie du sujet écrivant, on comprend que le personnage est surtout prétexte à dénonciation.

Slash évoque le parcours d'un jeune Okanagan qui cherche à sortir du marasme, désigné avec force par l'auteure grâce à cet oxymore «living death » (ARMSTRONG 1985, Préface 9). Slash est le témoin vivant de sa société qui se meurt à petit feu. Il fait l'expérience de l'alcool, de la drogue et de la prison. Il s'isole et se retrouve seul: "Most of the other prisoners kind of left me alone » (ARMSTRONG 1985, 72). Ce repli passager sur lui-même l'aide à prendre du recul. Jeune, il observe son entourage et essaie de comprendre ses pairs et prend comme exemples son père et son oncle qui restent fidèles à leurs valeurs, sous forme de résistance passive : «they were neither assimilated nor lost » (ARMSTRONG 1985, 70) et s'il les rejette dans un premier temps, il traverse plusieurs étapes: le retour vers des valeurs spirituelles et la résistance à l'assimilation. Le personnage de Slash, jeune adolescent, comprend déjà que la société dans laquelle il vit cherche à effacer ce qui fait l'unité de la Nation okanagane : «the white people wished we would either be just like them or stay out of sight ». (ARMSTRONG 1985, 36). La tristesse laisse place à la colère et Slash devient un meneur, un véritable tribun. 


\section{LA « TROISIÈME SOLITUDE » DU CANADA VÉCUE ET TRANSCRITE PAR DEUX ÉCRIVAINES AUTOCHTONES : JEANNETTE ARMSTRONG ET MARIA CAMPBELL}

La solitude de Maria Campbell est due à sa double appartenance biologique et culturelle. Issue d'une famille de Métis, elle est tiraillée entre la culture européenne, qu'elle a héritée de sa mère - «I grew up on Shakespeare, Dickens... » (CAMPBELL 1973, 17) - et la culture crie, transmise par son arrière-grand-mère paternelle, Cheechum.

Dans sa recension de l'article de Toni Cujak, Tai Grauman évoque le besoin pour l'individu de faire partie d'un groupe : «For Campbell, who was raised in a traditional Métis community, finding her identity was about discovering a sense of solidarity with her people » (2015). Elle souligne aussi la difficulté inhérente au statut de Métis :

Culjak helps us as readers understand how Métis culture is underrepresented within Canadian society. A theme [...] is the dichotomy of having to choose whether to assimilate to "White" culture or to become 'Indian' [...] the sense of being stuck in a place of "in-between", of being neither here nor there. (GRAUMAN 2015)

L'écrivain franco-libanais Amin Maalouf exprime la complexité des sentiments d'un «sang-mêlé » et les conflits internes liés aux besoins d'appartenance collective : «Ce qui fait que je suis moi-même et pas un autre, c'est que je suis à la lisière de deux pays... Serais-je plus authentique si je m'amputais d'une partie de moi-même ? » (1998, 5). Quant à Claude Grunitzky, Franco-Polono-Togolais, il se définit comme bénéficiant d'un héritage transculturel et porteur d'une identité hybride (2008).

C'est ce concept que l'on retrouve chez Kristina Frigan. Elle écrit qu'on ne sait plus très bien qui sont les siens. Un mouvement théorique connu sous le nom de «réalisme post-positiviste » peut offrir un moyen de comprendre les multiples identités exprimées dans le roman : "In fact, Campbell herself has commented that her work is about group identity: "it's not my story I'm telling; it's the story of a people" » (FRIGAN 2009, 259). Kristina Frigan voit en Maria Campbell une personne d'origines variées, un exemple d'identité fluide, hybride : « Hybridity theorists emphasize the ability of cultures to blend. Aboriginal people of mixed-descent are especially popular subjects for those interested in hybrid identity. Jodi Lundgren, for instance, views the Metis as inherently hybrid» (FRIGAN 2009, 262).

Mais à l'époque où Maria Campbell grandit (elle a vingt ans dans les années 1960), le métissage n'est pas à l'honneur. À la recherche de ses racines, souffrant d'un véritable déchirement entre deux cultures, la Métisse est assimilée 


\section{ISABELLE ELOY-CARRIAT}

à la femme autochtone et est victime du préjugé blanc : les Autochtones n'ont pas de culture. Ainsi confrontée au rejet des Blancs, elle revendique plutôt ses origines autochtones. Mais rien n'est fait pour que la femme métisse puisse s'affirmer de quelque manière que ce soit, et tout d'abord sur le plan juridique. De 1869 jusqu'à 1985, la Loi sur les Indiens institue une discrimination contre les femmes autochtones qui perdent leurs droits ancestraux et issus de traités si elles épousent un partenaire n'ayant pas le statut d'Indien. Maria Campbell, dans les années 1970 et 1980, rejoint la lutte pour la révocation de cette clause et revendique un «statut» pour les Indiennes non-inscrites. La modification de 1985 permet aux femmes mariées à des non-Autochtones et à celles qui, pour toute autre raison, ont perdu leur statut d'Indienne, de demander le rétablissement de leur statut et de leurs droits. Le cas du métissage est donc ainsi occulté en Amérique du Nord. Le vocable «half-breed»a un sens péjoratif; aucune reconnaissance juridique ne lui est accordée.

Affecté par l'image négative de la culture autochtone, l'enfant métis cherche bien souvent à nier ses origines et à rechercher l'assimilation, l'intégration à la société canadienne afin de bannir sa solitude, mais rejeté par son ascendance européenne, il trouve refuge parmi les Autochtones. Ainsi, Maria Campbell apprend de Cheechum à ne pas mépriser son peuple mais à honorer ses ancêtres et à ne plus éprouver de honte par rapport aux Blancs. Elle s'attache alors à défendre le peuple autochtone dont elle est issue en partie

Ce qui peut être perçu comme une force est aussi source de souffrance. De même l'enfant métis, dans un premier temps, doit nécessairement se construire à travers un groupe qu'il aura choisi au détriment des autres. Nelly Schmidt revient sur les origines de ce terme :

Le métissage humain apparu dès les premiers contacts entre groupes ethniques, fut lié, beaucoup plus tard, aux processus de conquête, de colonisation, de traite négrière [...]. Né d'une union longtemps considérée comme illicite [...] le métis fut très tôt prisonnier de préjugés aussi pesants que contradictoires [...] sans cesse accusé de duplicité (SCHMIDT 2003, 7).

L'auteure rappelle la leçon première de la génétique donnée dans Éloge de la différence (JACQUARD 1978) selon laquelle :

Les individus, tous différents, ne peuvent être classés, évalués, ordonnés : la définition de 'races', utile pour certaines recherches, ne peut être qu'arbitraire et imprécise; l'interrogation sur le 'moins bon' et le 'meilleur' est sans réponse (SCHMIDT 2003, 7) 


\section{LA « TROISIÈME SOLITUDE » DU CANADA VÉCUE ET TRANSCRITE PAR DEUX ÉCRIVAINES AUTOCHTONES : JEANNETTE ARMSTRONG ET MARIA CAMPBELL}

Enfant, Maria Campbell n'appartient ni à la société blanche ni à la communauté autochtone et elle vit cela comme un demi-statut. La communauté autochtone n'a rien d'attirant à ses yeux. Elle explique que les Autochtones sont souvent pauvres, oisifs et ont la réputation d'être des voleurs et des alcooliques (tant les hommes que les femmes). Ce modèle est rejeté par l'enfant métisse qui a le désir de s'identifier au «Blanc» dans un premier temps. Elle est attirée par les poudres et les parfums des Blanches. Elle a honte de faire partie des « havinots » (comme elle les nomme) et elle souffre de l'ambivalence qui consiste être à la fois admirée et rejetée par les Autochtones de par sa singularité physique : elle a les yeux bleus, héritage criant du Blanc : «the devil Scot».

Elle se sent rejetée, même au sein de l'Église catholique, et fait preuve d'incompréhension face à la bonne volonté montrée par les Métis : «In general the Halfbreeds were good Catholics » (CAMPBELL 1973, 31). Lorsqu'ils veulent intégrer leurs instruments et leurs chants à la cérémonie religieuse, ils sont renvoyés de l'église, paradoxe aux yeux de Maria: n'a-t-on pas voulu les « civiliser », les évangéliser ? L’Église catholique ne prône-t-elle pas tolérance et ouverture?

À cette époque, Métis et Autochtones évangélisés n’appartiennent pas à la même communauté religieuse : «Sandy Lake Reserve, which was a Church of England stronghold »(CAMPBELL 1973, 32). Très vite, Maria va être attirée davantage par le sacré que lui inculque Cheechum, avec toutes les croyances qui s'y rattachent et qu'elle appelle «The Great Spirit » : « Her philosophy was much more practical, soothing and exciting ... she taught me to see beauty in all things around me » (CAMPBELL 1973, 72). Maria arbore alors la tenue et la coiffure des autochtones (tresses), elle s'intéresse au pow-wow et à la médecine autochtone.

Lors de son séjour dans un pensionnat indien tenu par des Sœurs, elle vit une expérience traumatisante lorsqu'elle se retrouve enfermée dans un placard pour avoir parlé sa langue natale, le Cri. Elle dénonce le peu de savoir qui leur est inculqué par rapport aux heures passées à prier ou à effectuer des tâches ménagères. Plus tard, elle souffre de la ségrégation qui règne au sein même de l'école mixte construite près des siens et où elle se rend dès ses neuf ans: les Blancs sont assis d'un côté, les Métis, dont la plupart ignorent l'alphabet ou la base des mathématiques, de l'autre. Les Métis endurent moqueries et humiliation de leurs camarades d'école qui les surnomment «Road Allowance people» (personnes qui reçoivent des aides sociales), ce qui provoque chez Maria un fort ressentiment vis-à-vis de ses parents. Or Cheechum lui explique que les Blancs vont tout faire pour lui faire détester les siens : ce rejet des Métis, c'est une arme 


\section{ISABELLE ELOY-CARRIAT}

pour eux, et elle lui donne sa première leçon de vie : «stop fighting your parents and do something about it yourself » (CAMPBELL 1973, 47).

Du haut de ses douze ans, après le décès de sa mère, et avec les absences répétées de son père, elle doit assumer leurs rôles auprès de ses sept frères et sœurs. Ensuite, toute jeune mariée (quinze ans), elle subit le machisme de son époux métis : la femme à la maison avec les enfants, l'homme peut se distraire : il joue et s'adonne à la boisson... Maria exprime son amertume : «I had married to escape from what I'd thought was an ugly world, only to find a worse one » (CAMPBELL 1973, 116). Afin de subvenir à ses besoins et à ceux de sa fille Lisa, Maria va se prostituer. Elle fait aussi l'expérience de l'héroïne pour oublier ses origines et ses peines. Elle réussit à décrocher mais se met à boire et veut attenter à ses jours quand elle se sait enceinte de son deuxième enfant. Elle est sauvée grâce à des rencontres humaines heureuses ; elle décide alors, à son tour, d'aider les autres. En réaction à ses souffrances passées, la femme qu'est devenue Maria lutte contre les méfaits de l'alcool chez les Autochtones et elle s'engage pour améliorer leur sort : elle devient «Representative of the Metis Association of Alberta to speak to the Women's Section of Fort Saskatchewan jail » (CAMPBELL 1973, 132).

L'écrivaine utilise la métaphore de la couverture - fil rouge du récit qui lui vient de son arrière-grand-mère paternelle. Selon Cheechum, une fois que le gouvernement a tout pris (fierté, dignité) il vous donne une couverture : « they give you a blanket to cover your shame... all people wore blankets...not just Halfbreeds and Indians »(CAMPBELL 1973, 137). Elle insinue que personne n'est à l'abri. Maria revendique une place à part pour les Métis : «I said I was not a Treaty Indian but a Halfbreed » et elle est révoltée par le mépris et l'ignorance d'un employé des Services Sociaux qui lui répond : «'I can't see the difference part Indian, all Indian. You're all the same » (CAMPBELL 1973, 133).

Grâce à des prises de position similaires, les Métis sont aujourd'hui reconnus comme un peuple autochtone aux côtés des Premières Nations.

\section{Le militantisme vécu et relaté pour renforcer les liens de sa communauté}

Maria Campbell et Jeannette Armstrong ont traversé diverses expériences qu'elles décrivent avec une profonde sincérité, dans Halfbreed et Slash. Elles témoignent de leur frustration et de leur colère et expliquent comment elles s'impliquent dans l'activisme pour enfin parvenir à une affirmation de soi en tant qu'individu en relation étroite avec sa communauté. Mais quelle peut être la portée de ces textes sur les communautés auxquelles appartiennent leurs 


\section{LA « TROISIÈME SOLITUDE » DU CANADA VÉCUE ET TRANSCRITE PAR DEUX ÉCRIVAINES AUTOCHTONES : JEANNETTE ARMSTRONG ET MARIA CAMPBELL}

auteures? La question en effet se pose de savoir quel est le lectorat visé par les deux écrivaines, et quel est leur lectorat effectif.

Comme le fait remarquer K. Swathi, dès le début de son récit, Maria Campbell se montre très claire sur ses intentions, précisant qu'elle s'adresse à un lectorat euro-canadien blanc $(2018,18)$ : «I write this for all of you, to tell you what it is like to be a half-breed woman in our country. I want to tell you about the joys and sorrows, the oppressing poverty, the frustrations and dreams » (CAMPBELL 1973, 8). Il faut y voir un acte politique, un acte de résistance à la posture colonialiste de son époque, un acte avant-gardiste, comme le souligne Kristina Frigan : « As early as 1976, Beth Paul, in a CBC Radio documentary, described Halfbreed as the harbinger of 'A New Native Literature' that was 'informing non-Indians about us [Native people] as people' » $(2009,260)$.

En revanche, Helen Hoy est convaincue que Jeannette Armstrong s'adresse avant tout à un lectorat autochtone : «Slash was written to convey the spirit of 1960s and 1970s Native movement to the First Nations readers by tracing the emotional, political and spiritual turmoil of a young Okanagan man, Tommy Kelasket or Slash » $(2001,32)$. Helen Hoy s'intéresse à la réception d'un tel ouvrage chez un lectorat euro-canadien blanc. Elle explore les problèmes soulevés par la lecture d'auteures autochtones depuis la perspective d'un lecteur extérieur et distinct de par sa culture. En effet, le récit de Jeannette Armstrong ne répond pas à la norme occidentale en littérature; il faut donc le lecteur fasse un effort pour se mettre dans une position ouverte, pour vraiment comprendre, audelà du simple acte de lecture ou d'écoute: "The novel itself, though, is sufficiently inaccessible for many Western readers as to make glib professions of cross-cultural sensitivity difficult » (HOY 2001, 34).

Ce qu'Helen Hoy propose, face à ces difficultés d'ordre esthétique, est : « 'the intrinsic approach' called for by Armstrong and Lee Maracle (LUTZ, 29), what Jamaican novelist and critic Michelle Cliff calls 'reading from the inside out' ». (HOY 2001, 34). Elle fait appel à la notion de «cultural relativity » et s'appuie sur Lee Maracle, qui oppose «European literature, which aims to captivate from beginning to end », à « oral stories, Slash allowing the hearer to reflect on the truth unfolding » (HOY 2001, 38).

Le militantisme par essence aide à briser la solitude et à alléger le sentiment d'exclusion, aussi par leurs revendications, ces écrivaines tentent-elles de faire évoluer la condition d'autochtone et de femme, d'obtenir la reconnaissance de l'Autre et son inclusion dans la société malgré des « limites », au sens littéral comme au sens figuré. Les motivations qui poussent à écrire ou 


\section{ISABELLE ELOY-CARRIAT}

bien à s'engager politiquement ne sont-elles pas toujours plus complexes que celles que l'on veut bien reconnaître, et ce d'autant qu'elles ne sont pas forcément conscientes?

Dans Slash, Jeannette Armstrong retrace la lutte des Autochtones au Canada et aux États-Unis à la fin des années 1960 et au début des années 1970, avec la naissance du American Indian Movement, les modifications de la Loi sur les Indiens au Canada, la prise de contrôle du Département des Affaires indiennes au Canada et du Bureau of Indian Affairs aux États-Unis, et l'occupation de Wounded Knee.

La rébellion est devenue pacifique, une non-violence prônée par la majorité des femmes après l'échec des actions menées durant les années 1970 et 1980. Les Autochtones vont enfin sortir de leur isolement. En se joignant aux mouvements qui ont cours aux États-Unis, ils se rendent compte que leurs revendications sont identiques et qu'ils ont finalement les mêmes racines: «Indians from Canada were no different and [that] there was no real border that was recognized by Indians... we had the same objectives as U.S. Indians » (ARMSTRONG 1985, 92). Ils parviennent par exemple à des accords défendant la reconnaissance des langues autochtones, facteur majeur d'identité.

Maria Campbell pointe la difficulté de la femme autochtone, malgré son engagement politique et associatif, à se faire entendre parmi les siens. Dans de petits groupes informels ainsi que de grandes conférences auxquelles les femmes participent par milliers, elle s'attaque aux problèmes des pensionnats, des femmes tuées ou portées disparues, aux comportements violents et à l'abus de drogues, alcool et autres substances. Maria Campbell s'affirme comme « a young idealistic Native Woman » (CAMPBELL 1973, 145). Elle n'en subit pas moins une forme de machisme de la part de leaders autochtones qu'elle n'hésite pas à dénoncer : «When I too began to speak out, his attitude towards me changed ... I've met many Native leaders who have treated me the same ». Selon elle, l'Église a sa part de responsabilité : "The missionaries had impressed upon us the feeling that women were a source of evil » (CAMPBELL 1973, 144). Lorsqu'elle assiste à des meetings pour la défense des Autochtones, elle fait le constat suivant: «Women were not encouraged to attend unless a secretary was needed » (CAMPBELL 1973, 155).

Lorsqu'on lit Slash et de Halfbreed, l'énergie salvatrice des deux antihéros fait écho à la "résilience », concept élaboré par Boris Cyrulnik (2012). À partir des sentiments de souffrance et d'isolement, l'être humain peut trouver la force intérieure de se réaliser. Cela peut prendre la forme d'une survie à travers 


\section{LA « TROISIÈME SOLITUDE » DU CANADA VÉCUE ET TRANSCRITE PAR DEUX ÉCRIVAINES AUTOCHTONES : JEANNETTE ARMSTRONG ET MARIA CAMPBELL}

la communion avec les siens, d'une lutte commune. Chez Maria Campbell, le sentiment de solitude est si intense, qu'elle doit réagir, au risque de sombrer.

La créativité artistique devient aussi un exutoire et une thérapie que l'on peut résumer par l'art-thérapie : «I did a lot of art work and things, just to get my mind off hard feelings that bothered me about all that kind of stuff » (ARMSTRONG 1985, 49). Jeannette Armstrong, tout comme son personnage, a une prédilection pour les beaux-arts et elle a aussi contribué à l'écriture créative tout au long de sa carrière.

C'est souvent avec l'émergence d'un premier témoignage (l'autobiographie), et implicitement l'affirmation de leurs racines autochtones que ces écrivaines résistent. Écrire permet de s'affirmer et de se reconstruire grâce au genre autobiographique : conventionnel et classique, c'est-à-dire je réel / je de l'auteure $=$ je subjectif/narrateur (LEJEUNE 1975) avec Halfbreed, ou bien autobiographie fictionnelle, récit autobiographique à travers le filtre d'un protagoniste masculin dans Slash, récit qui se présente comme une fiction (voir le prologue de Slash) mais avec cette constante d'un narrateur s'exprimant à la première personne et témoignant de la vie intime de l'héroïne / auteure. Le Je réel/auteure est différent du Je fictif/narrateur (SCHMITT 2010). Il s'agit ici d'un bildungsroman (le lecteur suit la formation intellectuelle et morale du protagoniste) : on le voit lutter contre des pressions sociales et psychologiques afin d'affirmer de façon positive son identité et son appartenance à une communauté autochtone dans un monde qui évolue très rapidement autour de lui.

Maria Campbell, écrivaine militante, tente de résoudre quant à elle ses problèmes d'identité, de personnalité dédoublée malgré toute la complexité de cette entreprise. Son autobiographie s'avère utile à plusieurs degrés : d'abord, c'est un témoignage de ses actions concrètes de militante ; ensuite, par le biais de l'autobiographie, elle peut dénoncer les travers de la société canadienne ; enfin, ce récit lui offre la possibilité de rejoindre la sphère des écrivaines canadiennes engagées, signant ainsi un acte politique et littéraire. On peut se demander en l'occurrence si agir est plus efficace que l'acte d'écrire.

Le fait d'être marginalisée incite la femme autochtone à écrire un texte autobiographique, à cerner son identité et à revendiquer le droit à la culture autochtone ou métisse. Le lecteur assiste donc à l'évolution du témoignage qui de personnel, s'élargit en témoignage collectif.

Slash, quant à lui, se sent renaître, il appréhende la réalité de ce que signifie être un autochtone. Et bien que le protagoniste dans Slash soit un homme, 


\section{ISABELLE ELOY-CARRIAT}

ce roman est féministe. Slash déclare : «It's really the women who keep things going smooth [...] We learned early from our mothers and grandmothers that it is women who are the strength of the people » (ARMSTRONG 1985, 153).

\section{Importance de la trace écrite pour les générations futures}

Margery Fee s'intéresse à la démarche d'auteurs autochtones qui viennent offrir à leurs lecteurs un discours différent de celui issu de la colonisation. Maria Campbell tout comme Jeannette Armstrong répondent à ces critères :

The model of colonization has been applied to white-indigenous relations in Canada at the social and economic levels [...], but it works at the ideological and literary levels as well [...]. To decentre this model is not easy; however, indigenous activists have made progress in forming a collectivity strong enough to promote its own interests and have been actively constructing a counter-discourse [...]. Those that put the issue on the table, at least in literary terms, were Maria Campbell's Halfbreed. [...] More recent works are not so much aimed at educating white audiences as at strengthening Native readers' sense that there must be a better way to think about themselves than that presented by the dominant discourse. (FEE 1990,169)

Et les deux écrivaines ont décidé que leur narration se ferait à la première personne du singulier, « je » pour ne pas exclure l'Autre :

The use of the first-person narrator in each novel is a primary tactic used against the dominant discourse. [...] to present as a whole that which has always been seen as fragmented, the construction of a Native "I" performs a shift from that which has always been constituted as Other to Self. [...] Native readers finally will find what white Canadians take for granted a first-person voice that does not implicitly exclude them. (FEE 1990, 171)

Si écrire à la première personne offre le pouvoir de s'exprimer en son nom propre, Fee nous éclaire sur le fait que Jeannette Armstrong donne aussi la voix à des personnages dont l'opinion diffère de son personnage principal : «The novels give a voice directly to those who generally are silenced. [...] Slash consists not only of Slash's first-person narrative but also of the direct speech of many different characters, most of them disagreeing with Slash » (FEE 1990, 172).

Maria Campbell est consciente qu'elle va sortir de l'isolement par le biais de l'écriture : «Kate Vangen, for example, quotes Campbell saying 'I tried 


\section{LA « TROISIÈME SOLITUDE » DU CANADA VÉCUE ET TRANSCRITE PAR DEUX ÉCRIVAINES AUTOCHTONES : JEANNETTE ARMSTRONG ET MARIA CAMPBELL}

being the militant speaker and the activist.... That's when I realized that...writing was the best way to reach people' ». (FRIGAN 2009, 265).

Chez les Autochtones, l'accès à l'écriture a été postérieur à l'éducation dans les écoles religieuses où l'anglais était enseigné. Pour l'Autochtone, le défi est double : apprentissage d'une autre langue, puis de l'écriture. Au fil des ans, les anciens acceptent de passer d'une transmission orale à une transmission écrite, seul moyen moderne (avant Internet) d'inculquer un savoir aux jeunes générations d'Autochtones. S'il est en anglais, ce savoir est alors accessible à la société canadienne dans son ensemble. Dans les années 1970, l'écriture devient une arme pour lutter contre la situation d'infériorité dans laquelle est placée l'Autochtone et parvenir à convaincre les Blancs de s'intéresser à la culture indienne. L'écriture œuvre dans une certaine mesure à la transmission et la sauvegarde de cette culture méprisée. Et c'est par un récit écrit que Maria Campbell et Jeannette Armstrong ont décidé de témoigner de leur vie.

En outre, ces deux femmes écrivaines manifestent le désir de diffuser leur culture aux générations futures en s'adressant particulièrement aux enfants et en écrivant des livres spécifiquement pour eux. Les jeunes générations d'Autochtones doivent garder à l'esprit deux aspects liés à leur identité : la fierté d'être Autochtone et la nécessité de la survie du groupe. Ainsi, Maria Campbell, deux ans après s'être consacrée à son autobiographie, écrit son premier livre pour enfants, People of the Buffalo (1975); quatre autres œuvres suivront. Les premiers écrits publiés par Jeannette Armstrong, qui a par ailleurs œuvré à l'amélioration de l'éducation des Autochtones, sont des livres pour enfants, dès 1982 avec Enwisteetkwa (Walk in Water).

De nos jours l'héritage de la culture autochtone est susceptible de passer par le biais d'autres médias (photos, films, documentaires) mais ces derniers n'ont pas supplanté l'écriture.

Nombreux sont les critiques littéraires, à reconnaître l'influence majeure que Maria Campbell a eu sur les générations suivantes :

The movement initiated by Maria Campbell's Halfbreed is growing ever stronger" as, for many Aboriginal writers, Halfbreed is a "vehicle" for their own resistance and empowerment. And Campbell's autobiography continues to influence writers even decades after its publication. (SWATHI 2018, 18) 


\section{ISABELLE ELOY-CARRIAT}

Pour Kristina Frigan, l'influence de Campbell est indéniable parce que son œuvre fait sens : elle crée du lien et a réussi à atteindre un public bien plus large qu'escompté :

Daniel David Moses uses a metaphor of kinship to assert that Aboriginal writers in Canada experience Campbell as an active influence, calling her "[t]he mother of us all" and Lenore Keeshig-Tobias agrees, "[s]he is. Of course she is" (83). For Aboriginal writers, this sense of connection seems to come from a shared activity and purpose with Campbell. And this sense of connection is not unique to Metis readers; many of the Aboriginal writers and critics who were interviewed by Harmut Lutz in the early nineties also allude to the strong influence and connective quality of Halfbreed. (FRIGAN 2009, 267).

Hartmut Lutz souligne l'influence de Maria Campbell avec ces mots :

Of all the autobiographies written and published by Native individuals in Canada Campbell's is probably internationally the best-known and it has served as a model for later Native authors who are encouraged to follow the example set by the Metis women from Saskatchewan. (LUTZ 1991, 116)

Sans avoir pu même anticiper son impact sur le lectorat autochtone, Maria Campbell a été source d'inspiration pour des écrivains tel Gregory Scofield, Métis aux origines cries et qui a commencé à écrire vingt ans après la publication de Halfbreed: "Scofield asserts that writers such as Campbell 'made me want to write' » (FRIGAN 2009, 267).

\section{Conclusion}

Femme autochtone au sein de deux cultures, comment trouver ou retrouver le sentiment d'appartenance à une communauté minoritaire, elle-même intégrée à une communauté majoritaire, mais qui n'apparaît plus aussi dominante car elle est devenue moins opprimante ? Jeannette Armstrong et Maria Campbell ont trouvé une forme de thérapie pour remédier à leur solitude sous la forme de l'attachement à un mouvement unitaire. Elles ont eu à cœur d'œuvrer pour la réconciliation de tout un peuple d'abord avec lui-même et dans un second temps avec la société d'origine européenne.

La lutte pour les droits d'un peuple et pour les droits de la femme autochtone se rejoignent. De même, à travers leur parcours initiatique, ces deux écrivaines cherchent à découvrir leur identité personnelle et l'identité collective de leur peuple, ce qui confère à leurs ouvrages un aspect didactique. Bien qu'il 


\section{LA « TROISIÈME SOLITUDE » DU CANADA VÉCUE ET TRANSCRITE PAR DEUX ÉCRIVAINES AUTOCHTONES : JEANNETTE ARMSTRONG ET MARIA CAMPBELL}

soit légitime d'interroger les limites de la démarche et du positionnement identitaire et militant chez chacune des écrivaines, on ne peut nier à Maria Campbell et Jeannette Armstrong le privilège d'avoir réussi à sortir de l'isolement en tant qu'individu et en tant qu'écrivaine. Mais Kristina Frigan salue surtout cette capacité qu'a la littérature autochtone à relier les Autochtones entre eux et cite Emma LaRoque, une universitaire canadienne métisse d'origine crie :

Aboriginal critics have often focused on the role that Halfbreed has played in connecting Aboriginal people to one another. Emma LaRoque, for example, a contemporary of Campbell, explains that for her the text is not about resistance but about connection: "I do not read Maria Campbell's Halfbreed - I experience it. Maria told a story, her story. She did not use the Métis as a vehicle for a worldview, a doctrine or even as a social protest. She simply told a story, and because it is authentic, it is my story too" (91). (FRIGAN 2009, 267)

L'héritage de leur culture est lié à l'identité, qui elle-même se transmet par la langue anglaise (parlée puis écrite). La littérature de la femme autochtone tient une place essentielle dans la promotion des valeurs autochtones et la transmission de l'héritage culturel.

Après la déchéance (due au désespoir, à l'alcool, à la drogue, à la prostitution) l'être humain peut chercher sa résurgence (grâce à l'activisme, à la littérature et aux activités artistiques) au prix de nombreuses souffrances, car la complexité de la condition humaine vient de la quête perpétuelle d'équilibre individuel et collectif.

Selon Helen Hoy, Jeannette Armstrong aussi bien que Maria Campbell, refusent d'être confinées dans des catégories identitaires de femme ou Autochtone, et elles fournissent une voix critique afin de guider la façon dont leurs œuvres doivent être lues et enseignées. Margery Fee met en exergue la question intrinsèque au récit de Maria Campbell, que l'écrivaine a énoncée ellemême : «Why can't I be me, with my bag of stuff, within what's supposed to be our society? » (FEE 1990, 178)

Pour Jeannette Armstrong, son peuple ne fait en aucun cas partie d'une troisième solitude; elle propose un «troisième choix »: « Assimilate or get lost. A lot of us are lost. We need to make a third choice » (ARMSTRONG 1985, 70). C'est le thème central de son livre, une autre vision d'un monde centré sur le passé et les traditions du peuple okanagan. 


\section{ISABELLE ELOY-CARRIAT}

\section{Bibliographie}

ARMSTRONG, Jeannette. 1985. Slash. Penticton, BC: Theytus Books.

AYLSWORTH, Laura et TROVATO Franck. 2012. Modification 2015. «Démographie des Autochtones ». https://www.thecanadianencyclopedia.ca/fr/article/demographie-desautochtones

CAMPBELL, Maria. 1973. Halfbreed. Toronto: McClelland and Stewart Limited.

CUJAK, Toni. 2001. "Searching For a Place in Between: The Autobiographies of Three Canadian Metis Women." The American Review of Canadian Studies 31(12): 137-157.

CYRULNIK, Boris. 2012. La Petite Sirène de Copenhague. Paris : Éditions de l'Aube.

FEE, Margery. 1990. "Upsetting Fake Ideas: Jeannette Armstrong's Slash and Beatrice Culleton's April Raintree." Canadian Literature 124-125: 168-180.

FRIGAN, Krisitina. 2009. "Reading the Reception of Maria Campbell's Halfbreed." The Canadian Journal of Native Studies 24(1-2): 257-281.

GRAUMAN, Tai. 2015. "Campbell compared to other autobiographies”. Review of Culjak, Toni A., "Searching For A Place In Between: The Autobiographies of Three Canadian Métis Women". August 10. https://blogs.ubc.ca/diasporasandinterventions/2015/08/10/campbell-comparedto-other-autobiographies/

GRUNITZKY, Claude. 2008. Trans culturalismes (essais, récits et entretiens). Paris : Grasset.

HOY, Helen. 2001. How Should I Read These? Native Women Writers in Canada. Toronto: University of Toronto Press.

HUNGRY WOLF, Beverly. 1982. The Ways of my Grandmothers. New York: Quill.

JACQUARD, Albert. 1978. Éloge de la différence. Paris : Le Seuil.

LEJEUNE, Philippe. 1975. Le Pacte autobiographique. Paris : Éditions du Seuil.

LUTZ, Hartmut. 1991. Contemporary Challenges: Conversations with Canadian 
LA « TROISIÈME SOLITUDE » DU CANADA VÉCUE ET TRANSCRITE PAR DEUX ÉCRIVAINES AUTOCHTONES : JEANNETTE ARMSTRONG ET MARIA CAMPBELL

Native Authors. Saskatoon, Saskatchewan: Fifth House Publishers.

SCHMIDT, Nelly. 2003. Histoire du métissage. Paris : Éditions de la Martinière.

SCHMITT, Arnault. 2010. Je réel, Je fictif. Toulouse : Presses Universitaires du Mirail.

STATISTIQUE CANADA. 2016. La population autochtone au Canada, Recensement de la population de 2016. https://www150.statcan.gc.ca/n1/pub/11627-m/11-627-m2017027-fra.htm

SWATHI, K. 2018. "A Subaltern Study of Maria Campbell's Half-Breed." Shanlax International Journal of English 6:17-20. 